\title{
The Various Types of Neurogenic Bladder Dysfunction: An Update of Current Therapeutic Concepts
}

\author{
H. Madersbacher, Prof Dr med \\ Department of Urology, University Hospital Innsbruck, Consultant Urologist, Rehabili- \\ tation Center Bad Häring, Tirol, Austria .
}

\section{Summary}

Increased experience with treatment strategies developed during the last 10 years in the field of neurourology justifies an update of current therapeutic concepts. Based on a rather simple, but clinically useful, classification of detrusor-sphincter dysfunction the therapeutic concepts now available for four prototypes of detrusor-sphincter dysfunction are discussed.

(1) For the combination of a hyperreflexive detrusor with a hyperreflexive (spastic) sphincter, characteristic for the reflex-and the uninhibited neuropathic bladder, detrusorsphincter dyssynergia (DSD) is still the greatest problem, and transurethral sphincterotomy is the method of choice if this situation cannot otherwise be managed. One concept is to convert detrusor hyperreflexia into hyporeflexia by adequate pharmacotherapy, which is nowadays available, and to assist or to accomplish bladder emptying by clean intermittent (self-) catheterisation (CIC) with the advantage of dry intervals in between. Fapanese colleagues recommend bladder overdistension during the spinal shock phase to achieve detrusor hyporeflexia, but this procedure is rather decisive at an early stage of the disability, leaving the detrusor no chance for further rehabilitation. Another possibility is rhizotomy of the sacral posterior roots to eliminate detrusor hyperreflexia, and the simultaneous implantation of a sacral anterior root stimulator (Brindley) to achieve electrically induced micturition. From our personal experience with 12 patients this concept is ideal for female patients with unbalanced reflex bladder and otherwise uncontrollable reflex incontinence.

(2) The combination of a weak detrusor with a spastic sphincter is a clear indication for CIC, as the bladder is emptied regularly, and due to the spastic sphincter, the patient stays continent as long as controlled fluid intake prohibits overflow incontinence. The implantation of an anterior sacral root stimulator is an alternative approach provided that at least weak reflex detrusor contractions are present.

(3) With the combination of an areflexive or hyporeflexive detrusor and a flaccid pelvic floor, passive voiding by abdominal straining or by the Crede manoeuvre is usually recommended, but should be replaced by CIC if this mechanism of bladder emptying creates unphysiological high and dangerous intravesical pressures, or if vesicouretero-renal reflux is present. Neurogenic urinary stress incontinence is usually associated with this type of lesion and can be successfully treated by the implantation of an artificial urinary sphincter (Scott). However in two thirds of the patients with neurogenic bladder 
dysfunction, additional, usually operative treatment is necessary to meet the criteria for implantation. Moreover, a 30\% rate of repair operations must be accepted by patients, but is becoming less frequently required with an improved design of the device. With conventional operations continence can only be achieved in those patients by maximal elevation of the bladder neck thus inducing bladder outflow obstruction requiring consequent CIC, but CIC may be difficult to perform due to the anatomical situation of a maximally elevated bladder neck.

(4) If a hyperreflexive detrusor is combined with a weak sphincter, again urinary incontinence is the greatest problem. To begin with, elimination of detrusor hyperreflexia is mandatory and can often only be accomplished by surgery, either by bladder augmentation using an isolated detubularised segment of ileum serving as energy destroyer, or by sacral posterior root rhizotomy. Sometimes the elimination of detrusor hyperreflexia in combination with CIC provides a socially acceptable situation, but neurogenic urinary stress incontinence has to be treated by an artificial urinary sphincter.

(5) Finally, in patients with incomplete spinal cord lesions intravesical electrotherapy (Katona, 1975), based on receptor depolarisation contributes greatly to the rehabilitation of the neuropathic bladder. In children with myelo- or sacral dysplasia this method together with biofeedback is an indispensible tool within a complex training programme for bladder rehabilitation.

The increased therapeutic armentarium is responsible for the changing concepts in the treatment of neuropathic detrusor and sphincter dysfunction, and moreover offers the possibility of choice for the same type of neuropathic bladder but with special respects for the individual needs of the patient. It should be stressed that adequate primary care is the prerequisite for successful application of these new treatment strategies and that functional and morphological changes do also occur with these types of therapy, and therefore lifelong urological care is necessary.

Key words: Spinal cord injuries; Neurogenic bladder dysfunction; Therapeutic concepts.

Lower urinary tract dysfunction resulting from spinal cord injury (SCI) is still responsible for a high morbidity, and for inconvenience such as urinary incontinence often limiting the patient's social activities. Proper urological care is a very important part of the complex treatment of the spinal cord injured.

Increasing experience with different treatment strategies, developed during the last 10 years in the field of neuro-urology, justifies an update of current therapeutic concepts. This report reflects our experience with some of these techniques.

\section{General remarks}

The tasks of the urinary bladder muscle and its sphincters are 2-fold: (1) the collection of urine with maintenance of urinary continence, which implies the capability of the detrusor to relax and of the sphincter mechanism to maintain the bladder outlet closed even with a sudden increase of intra-abdominal pressure, and (2) the emptying of the bladder at will, without residual urine, which implies the capability of the detrusor to contract and of the sphincter to relax simultaneously. This synergistic action is a prerequisite for complete emptying with a physiological intravesical pressure.

Damage to the innervation of the lower urinary tract mainly effects both the 
detrusor and the sphincter. For any therapeutic consideration the evaluation of the detrusor and the sphincter is essential.

\section{Classification of detrusor-sphincter dysfunction}

Various classifications have been used to define neurogenic lower urinary tract dysfunction (Krane and Siroky, 1979). The fact, that the evaluation of detrusor and sphincter is essential for any therapeutic consideration has led us to use a rather


NEUROGENE BLASENTYPEN
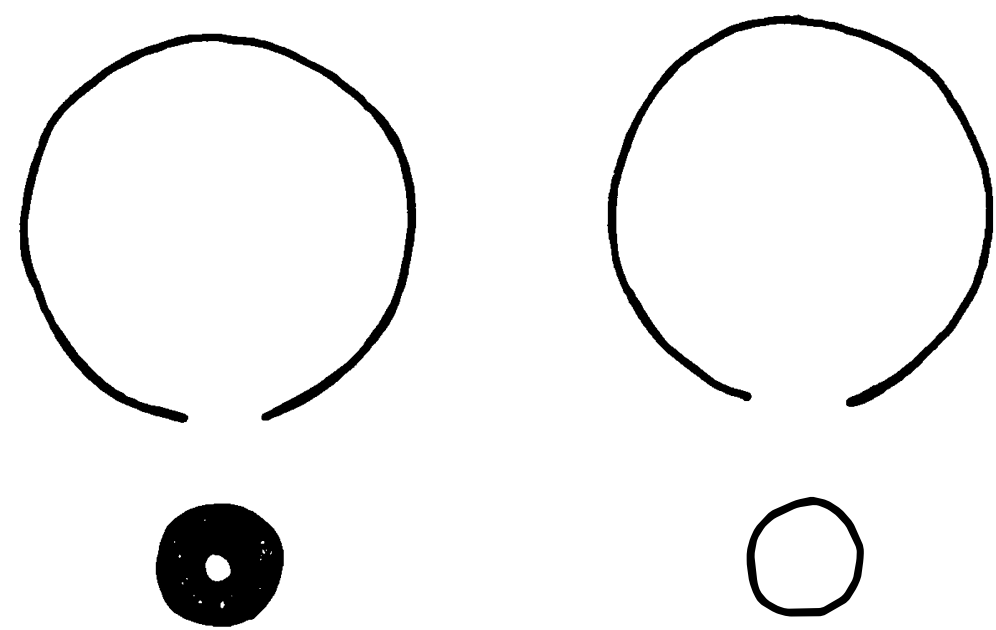

Figure 1 Each of the 4 schematic drawings represents a dysfunctional state of the detrusor-sphinctersystem; structures drawn in heavy lines are supposed to be hyperreflexive, those with thin lines to be hyporeflexive resp. areflexive (for further explanation see text).

simple but functional classification in clinical practice (Fig. 1). Each of the four schematic drawings represent a dysfunctional state of the detrusor-sphincter system. Detrusor and sphincter can be affected similarly, both being either 
hyperreflexive or both being hyporeflexive. Alternatively they are affected differently, either a combination of a hyporeflexive detrusor with a hyperreflexive sphincter or, a hyperreflexive detrusor with a hyporeflexive sphincter can be found. These four types of detrusor-sphincter dysfunction are those most commonly found in SCI. Each of these hyper- or hyporeflexive structures can theoretically be combined with a more or less normal counterpart, but these situations are rather rare in these patients. Although damage to the sensory pathways is not separately considered, this classification has proved to be extremely useful in defining detrusor-sphincter dysfunction and therefore provides the basis for further discussion on current therapeutic concepts.

Therapeutic considerations of the various types of neurogenic detrusorsphincter dysfunction

Detrusor hyperreflexia in combination with a hyperreflexive (spastic) sphincter

This combination is characteristic of the so-called reflex bladder, occurring in complete suprasacral lesions, and for the uninhibited neuropathic bladder as it is found in incomplete neurological lesions. In both, detrusor contractions are uncontrolled and inappropriate. Detrusor-sphincter dyssynergia (DSD) is another typical finding. Instead of sphincter relaxation during detrusor contraction, both contract at the same time. DSD is a dangerous phenomenon as it causes a functional obstruction with effects on the lower and the upper urinary tract, consequences which are best expressed by 'DSD kills kidneys'.

In this type of neurogenic detrusor-sphincter dysfunction both uncontrolled, sometimes hypercontractile and not sufficiently lasting detrusor contractions occur together with a functional outflow obstruction, mostly at the level of the external (striated) sphincter. This may result in unbalanced micturition with a high risk of upper urinary tract damage by reflux and dilatation and by urinary incontinence due to the lack of voluntary detrusor control. Bladder emptying is achieved either by spontaneous reflex contractions or by such a technique as suprapubic tapping. Compensated voiding without additional therapy is achieved in about 25 to $40 \%$ of patients, depending to a great extent on the quality of primary care. Regular urodynamic check-ups are essential. If risk factors indicate a dangerous situation or if secondary structural changes are already established, another therapeutic concept is mandatory.

The therapeutic strategy can be (1) to eliminate or diminish the functional outflow obstruction created by DSD or (2) to decrease detrusor hyperreflexia or (3) to treat both at the same time.

Elimination of functional outflow obstruction created by DSD. Adequate management of DSD is indicated, if it causes detrusor hypercontractility, vesicouretero-renal reflux, massive reflux into the prostate and if sphincter spasticity is the main reason for unbalanced voiding.

Drugs may help in some patients, but generally the effect of striated muscle relaxants such as Baclofen (Jones et al., 1970) is poor. The same is true for alphareceptor blocking agents such as Phenoxybenzamine, which relaxes the smooth 
muscle component of the sphincter, but the net effect on sphincter hyperreflexia clinically is not relevant.

Elimination of pudendal nerve activity was tried in the past (Ross and Damanski, 1953) but the side-effects such as loss of reflex erections and increased incontinence were unacceptable. A temporary, reversible, pharmacological 'sphincterotomy' (Mounzer et al., 1984) activated only for micturition, could be an alternative in the future.

To date, the method of choice at least to relieve severe striated spincter dyssynergia in males is transurethral sphincterotomy. The technique of anteromedian (12 o'clock) sphincterotomy, is comparatively simple, safe and very effective (Madersbacher and Scott, 1976). After sphincterotomy, balanced voiding with low detrusor pressure can be achieved. The disadvantages are that the operation is destructive and irreversible and that basically urinary incontinence persists although paradoxically this may be improved due to a decrease of detrusor hyperreflexia after elimination of the outflow obstruction. Sphincterotomy is now indicated if sphincter spasticity cannot be managed otherwise (see also below).

Elimination of detrusor hyperreflexia. The second possibility is to eliminate detrusor hyperreflexia pharmacologically. A number of potent smooth muscle relaxant agents with anticholinergic, direct spasmolytic and calcium blocking properties are available and may change detrusor hyperreflexia into hyporeflexia or even into areflexia (Fig. 2). The combination of a pharmacologically induced hyporeflexive detrusor with a hyperreflexive (spastic) striated sphincter, the function of which is not influenced by smooth muscle relaxants, usually results in unbalanced bladder emptying, which then has to be accomplished or assisted by intermittent (self-)catheterisation (CIC). The combination of a pharmacological conversion to a hyporeflexive detrusor together with a spastic sphincter usually provides dry periods between the catheterisations.

Intermittent catheterisation (Guttmann, 1947) has proved to be successful for bladder emptying during the spinal shock phase, but the safety of long term treatment when practised as CIC is now being assessed. Hill and Davies (1988) reported good results with this therapy in 234 patients. We are currently reviewing 57 patients on CIC for up to 34 years ( 1 for 34 years) with a mean observation of 11.8 years. According to the last follow-up, morphology and function of the upper urinary tract remained or has become normal in $83.5 \%$, has remained abnormal in $3.5 \%$, and became abnormal in $13 \%$; in half of them the changes were only mild. In 3 patients with vesico-uretero-renal reflux, renal damage occurred. From our experience vesico-uretero-renal reflux persisting with CIC should be corrected surgically in order to prevent recurrent urinary tract infections with consequent renal damage.

With new developments in catheter design and handsome catheter kits, CIC seems to be a safe procedure. We recommend catheterisation out of the sheath (Madersbacher and Weissteiner, 1978) using disposable catheters. However, the question remains, if and to what extent lifelong intake of anticholinergic drugs has consequences for other organ systems, e.g. for the gastrointestinal tract (atony of the stomach, increased constipation). The intravesical application of these drugs together with CIC may be a valuable alternative (Brendler et al., 1987; Jilg and Madersbacher, 1989). 
A
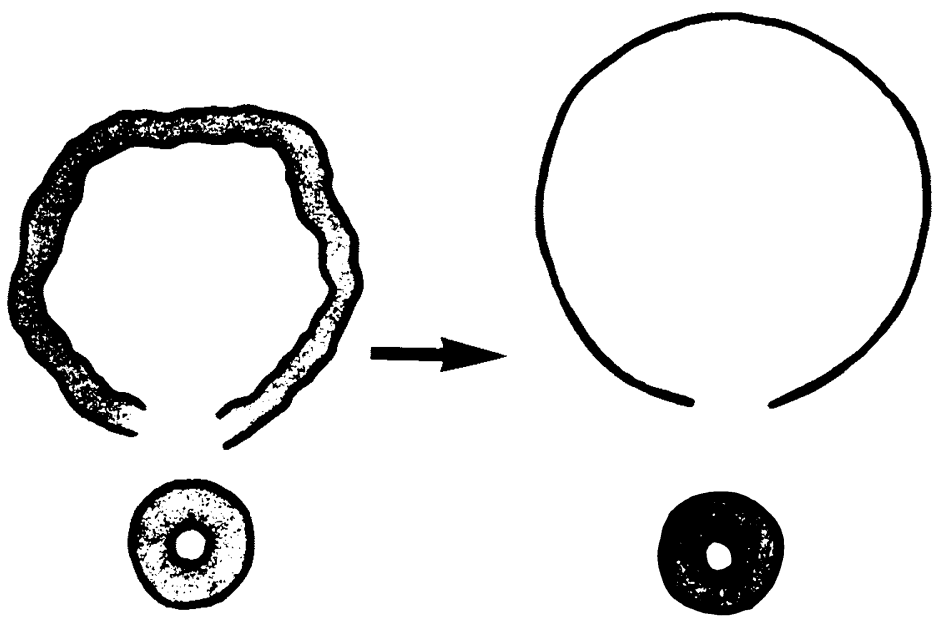

ST. J. 22 a - post-traumatic

spinal cord lesion, sub. D4/D5

2 February 1988
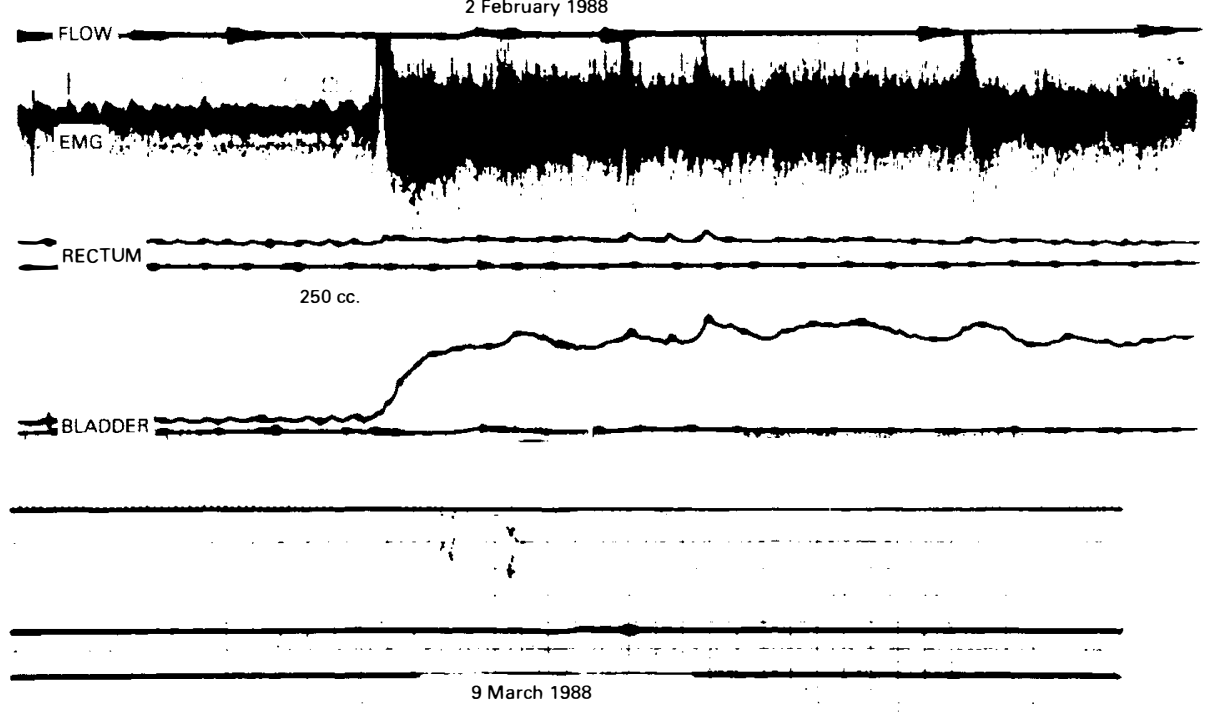

WITH TROSPIUMCHLORID

$2 \times 20 \mathrm{mg}$ daily



Figure 2 With the help of potent smooth muscle relaxant agents detrusor hyperreflexia can be changed into hyporeflexia or even into areflexia; bladder emptying can be accomplished or assisted by intermittent (self-)catheterisation. A Schema of this therapeutic concept. B Conversion of detrusorhyperreflexia into areflexia in a 22 -year-old paraplegic with Trospium chloride $20 \mathrm{mg}$ t.e.d. (Spasmolyt ${ }^{\circledR}$ ). Cystometry: intravesial and intrarectal pressure curves before (above) and after (below) the administration of the block. 
Recurrent overdistension of the bladder during the spinal shock phase is another way to achieve detrusor hyperreflexia (Iwatsubo et al., 1988) for female patients with complete suprasacral lesions. This approach is rather decisive at an early stage of the disability, leaving the detrusor no opportunity for further rehabilitation.

Rhizotomy of the relevant posterior sacral roots is another way to eliminate detrusor hyperreflexia, and with appropriate technique seems to yield, to date good and stable results (vide infra).

Elimination of detrusor hyperreflexia and achievement of balanced voiding despite sphincter spasticity. The aim to abolish detrusor hyperreflexia and to achieve balanced voiding despite sphincter spasticity at the same time can be achieved by rhizotomy of the sacral posterior roots and by simultaneous implantation of an anterior sacral root stimulator (Brindley, 1984). Interruption of the sacral reflex roots by rhizotomy abolishes the detrusor hyperreflexia, whereas effective micturition can be achieved by electrostimulation using the concept of post-stimulus voiding.

Our personal experience with this concept-rhizotomy of the posterior roots and implantation of an anterior sacral root stimulator-in 12 patients, 10 women and 2 men, with an observation time up to 3 years, is so far excellent. All the patients void by electromicturition, all but 1 male with a physiological detrusor pressure, and all are continent day and night. We consider that the ideal patient for this approach is a woman with an unbalanced reflex bladder and with incontinence due to uncontrollable (reflex-)detrusor contractions, which cannot be managed otherwise. Division of the sacral posterior roots in females results in loss

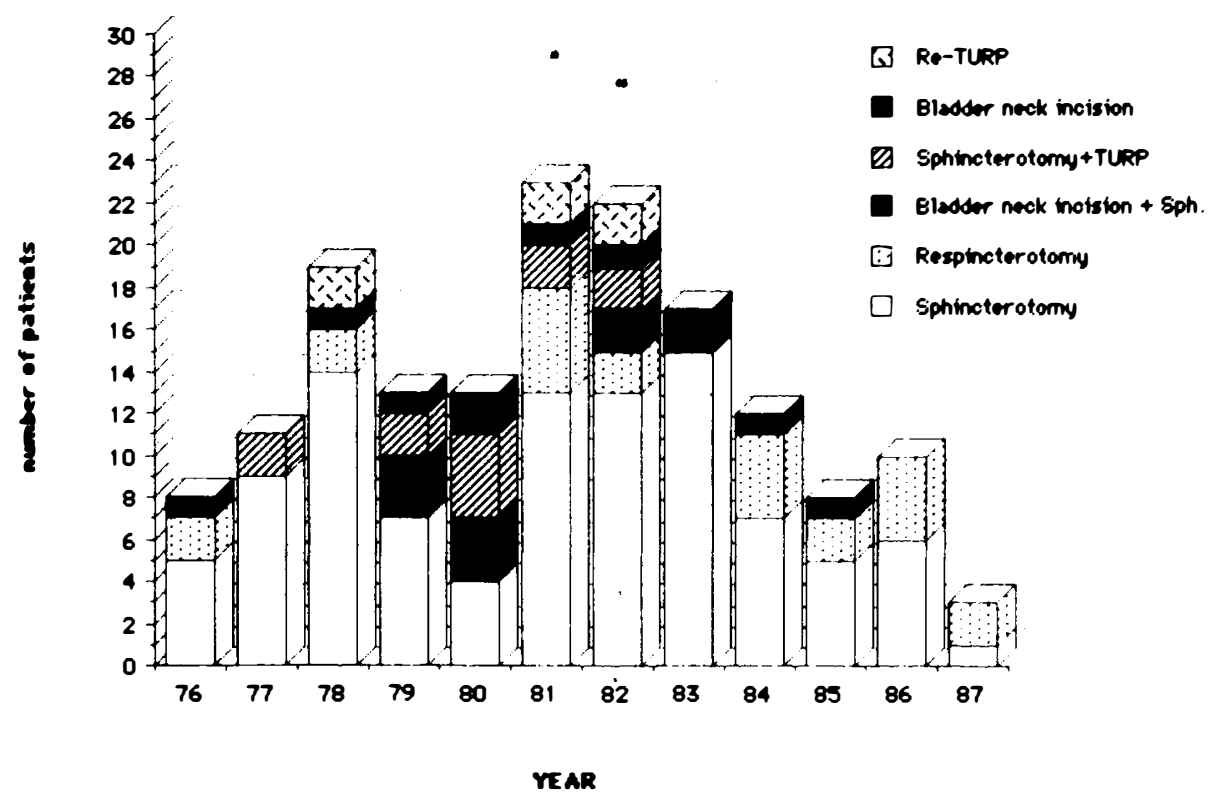

Figure 3 Survey of sphincterotomies and other transurethral operations to reduce outflow resistance in patients with spinal cord injuries between 1976 and 1987, performed at the Rehabilitation Center Bad Häring (Austrian Workers Compensation Board). Due to alternative treatment concepts the number of operations has decreased considerably within the last years. 
of vaginal lubrication and in males, loss of reflex erections. In male patients the posterior roots should only be divided in those who agree with the risk of loss of reflex erections from the procedure. Electro-erection can only be achieved in about $30 \%$.

Although some questions in regard to this treatment cannot yet be fully answered, e.g. whether posterior rhizotomy, which is so important to achieve continence, will last lifelong, this therapeutic approach is so far the only one which provides balanced voiding (by electromicturition) and urinary continence (by rhizotomy) at the same time. Electromicturition with low residual urine under physiological detrusor pressure definitively decreases the morbidity in these patients. Moreover rhizotomy, providing continence, makes life much easier for a spinal cord injured person.

In summary, various therapeutic concepts are at our disposal for the management of an unbalanced reflex bladder together with reflex urinary incontinence. Sphincterotomy, once the method of choice, at least in males, still has its indications, but only if the problem cannot be managed otherwise. Therefore the number of operations to relieve bladder outflow obstructed in patients with SCI has decreased considerably between 1976 and 1987 in our institutions (Fig. 3). This trend is the result of the good experience with CIC for long term management, the development of new and more effective spasmolytic drugs and of the possibility to implant an effective system for electromicturition.

\section{Detrusor hyporeflexia in combination with a hyperreflexive (spastic) sphincter}

With this combination of a weak detrusor and a spastic sphincter clean intermittent (self-)catheterisation is again the method of choice. The bladder is emptied regularly and the patients normally remain continent in between. If CIC is not possible for one or other reason, the implantation of a sacral anterior root stimulator (Brindley) may be considered if at least weak systolic detrusor contractions are present spontaneously or can be induced with transrectal electrostimulation, but additional sphincterotomy may be necessary in males.

Sphincterotomy alone is not advisable in this type of neuropathic bladder, as it usually does not provide balanced voiding. Even when combined with a bladderneck incision and/or a TUR of the prostate an additional Credé manoeuvre is necessary, with all the disadvantages that will be mentioned later.

On the other hand permanent urinary drainage either transurethrally or suprapubically should be avoided whenever possible. In the long term recurrent urinary tract infections cannot be avoided even when using a closed drainage system and infection prophylaxis. Permanent drainage combined with recurrent urinary tract infections often cause bladder shrinkage. Over the years this condition will inevitably result in a small, unelastic (low compliance) bladder and consequent upper urinary tract damage as well as urinary incontinence through the urethra around the transurethral catheter.

\section{Detrusor hyporeflexia (areflexia) in combination with a hyporeflexive (flaccid) sphincter}

With this type of detrusor-sphincter dysfunction, characteristic for conus-cauda lesions, usually passive voiding by abdominal straining or by the Credé-manoeuvre 
is recommended. Although the pelvic floor musculature may be totally flaccid often abdominal straining with an unphysiologically high intravesical pressure is necessary to empty the bladder. During passive voiding, the bladder and bladder outlet are pressed downwards and tipped backwards, while other parts of the urethra are fixed in the area of the symphysis. In this way the membranous urethra is compressed as it traverses the pelvic floor muscle giving an intravesical obstruction at the level of the pelvic floor (Madersbacher, 1977). The uncontrolled Credé manoeuvre (without knowing the intravesical pressure exerted) used over the years may result in vesico-uretero-renal reflux and consequent damage of the upper urinary tract. Especially in children due to the increasing mass of pelvic floor musculature an increasing force is needed for the Credé manoeuvre to empty the bladder. From our experiences there are significant contraindications for passive bladder emptying by the Credé technique and/or abdominal straining because of the necessity for non-physiological high intravesical pressure to evacuate the bladder, and the presence of vesico-uretero-renal reflux. With these conditions $\mathrm{CIC}$ is strongly recommended for bladder emptying.

Another problem associated with sphincter weakness is neurogenic urinary stress-incontinence. Regular bladder emptying with low or zero residual urine (e.g. by CIC), controlled fluid intake and treatment or prophylaxis of urinary tract infections will reduce neuropathic urinary incontinence in many patients to a socially acceptable degree. However, most patients do need appliances. Urinary incontinence can be managed in males, at least to obtain social acceptance with a condom-urinal, but for women the situation is worse, and wearing diapers, although improved in quality, is still an unsatisfactory alternative. In males, in whom a retractile penis impedes the condom style urinal, implantation of a (semi-rigid) penile prosthesis is advisable.

For selected patients the artificial urinary sphincter (Scott) is the alternative to provide continence. Proper patient selection is important; the key points of which are balanced voiding either spontaneously or assisted/accomplished by CIC, detrusor areflexia but if detrusor hyperreflexia is present this, as well as a vesicoureter renal reflux, must be controllable, together with motivation and manual dexterity. So far we have implanted the Scott-sphincter in 27 patients with pure neuropathic urinary incontinence. The overall results are satisfactory: 25 patients do use their sphincter, 24 are continent day and night; 2 patients are still stressincontinent. In 1 the cuff has become too wide and in the other the pressure regulation balloon is too weak. In one girl the cuff had to be removed because of perforation at the bladder neck. However, in 16 out of the 27 patients various operative procedures either before or during the implantation had to be performed to reach the criteria necessary for implantation. Moreover, $30 \%$ of these patients needed one or more repair operations, mostly due to mechanical failures mainly in systems of the earlier designs. So far the artificial urinary sphincter is the only way to achieve continence in neuropathic urinary stress incontinence without creating obstructive voiding.

The alternative, at least in female patients, is to perform a conventional colposuspension or sling procedure. However, in these patients the bladder neck has to be elevated maximally to achieve continence by mechanical occlusion, therefore, catheterisation for bladder emptying is essential but may be technically difficult or even impossible because of the upward dislocation of the bladder neck. 
The future will show if these procedures give good long term results (Schmidbauer et al., 1988; McGuire, 1967).

\section{Detrusor hyperreflexia in combination with sphincterhyporeflexia}

With this type of neuropathic bladder, which we see in about $15 \%$ of the children with myelomeningocele, urinary incontinence is severe and is the main problem. An active detrusor associated with passive sphincter weakness results in urinary incontinence, usually constant dribbling. The capacity of these bladders is governed entirely by the point at which either a reflex detrusor contraction or any other intravesical pressure increase goes beyond the pressure of low outflow resistance. The therapeutic concept must consider both the hyperreflexive detrusor as well as the weaker sphincter. In general, treatment of sphincter weakness alone in the presence of a hyperreflexive detrusor is dangerous. The first aim is to abolish detrusorhyperreflexia either pharmacologically, by posterior sacral root rhizotomy (which is not possible in children with myelomeningocele due to the anatomical situation) or by bladder augmentation using a detubularised segment of the gut, preferably ileum, as an energy destroyer. Our favourite procedure is the clam-ileocystoplasty, described by Bramble (1982) modified and popularised by Stephenson and Mundy (1985).

Depending on the degree of sphincter weakness sometimes control of detrusor hyperreflexia together with regular, balanced bladder emptying results in a socially acceptable situation; if not, the implantation of an artificial urinary sphincter is the method of choice (Fig. 4).

The indication for major bowel surgery such as a subtotal bladder substitution using ileum, coecum or both, should be very limited and should only be performed in women in whom uncontrollable urinary incontinence is caused by a shrunken bladder and/or subsequent progressive upper urinary tract deterioration is present. It should be the last step before a supravesical urinary diversion is considered.

In incomplete spinal cord lesions rehabilitation of detrusor function may be improved or at least speeded up by Katona's technique of intravesical electrotherapy by means of transurethral electrostimulation (Katona, 1958, 1975). For this purpose a special catheter with an electrode mounted on its tip is introduced in the bladder which is filled with saline as the current leading medium. Intravesical electrotherapy is based on the depolarisation of the specific receptors in the bladder wall thereby activating the intramural motor system. As soon as this local motor reaction reaches a certain strength, activation of the micturition reflex takes place. Electrically induced depolarisation of the receptors induces more and more forceful afferent and lastly also efferent stimuli inducing or reinforcing the micturition reflex. This goes along firstly with the induction or an improvement of the sensation of urge ('vegetative afferentation') followed by improved detrusor contractility (Fig. 5). Stimulation is carried out on a daily basis, for about 90 minutes, over weeks and months.

We have experience with this type of treatment especially in children with a congenital neuropathic bladder (Madersbacher, 1984), but also with a group of 80 adults mainly with post-traumatic incomplete spinal cord lesions. In this latter group in about one third of the patients the improvement or normalisation of detrusor function must be attributed to intravesical electrotherapy, in the sense 
$\mathbf{A}$

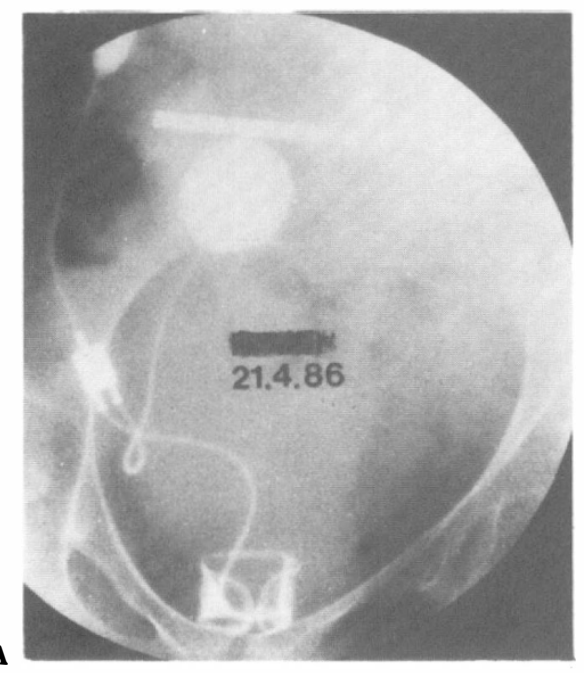

B



Figure 4 An 18-year-old female paraplegic with neurogenic bladder dysfunction after spinal cord injury with severe detrusor hyperreflexia combined with sphincter weakness. A clam-ileocystoplasty was performed for bladder augmentation; at the same time an artificial urinary sphincter was implanted (20.1.86), Xray-control (21.04.86). A Plain picture showing the implanted artificial sphincter system (model AMS 792). B The cystogram shows the augmented bladder, there is almost no difference in the outline between the bladder wall and the ileum patch. The sphincter system is closed, the patient is continent. C Voiding cystourethrogram, the sphincter is open (cuff deflated), voiding is accomplished by minimal abdominal straining.

that transurethral electrostimulation (TES) was responsible for the result. In others it may have at least speeded up bladder rehabilitation (Madersbacher, 1982, 1987). TES therefore is a useful help in the rehabilitation of neurogenic bladder dysfunction in patients with incomplete spinal cord lesions. The preservation of pain sensation in the sacral skin segments (especially in S3) indicates intact sacral pathways between the peripheral and the central nervous system, and is therefore a good prognostic sign for successful treatment. The underlying basis for this type of electrotherapy implies that patients with complete or almost complete lesions as well as those with irreversible damage of the receptors in the bladder wall because of recurrent urinary tract infections or recurrent overdistensions are not suitable for this therapy. 


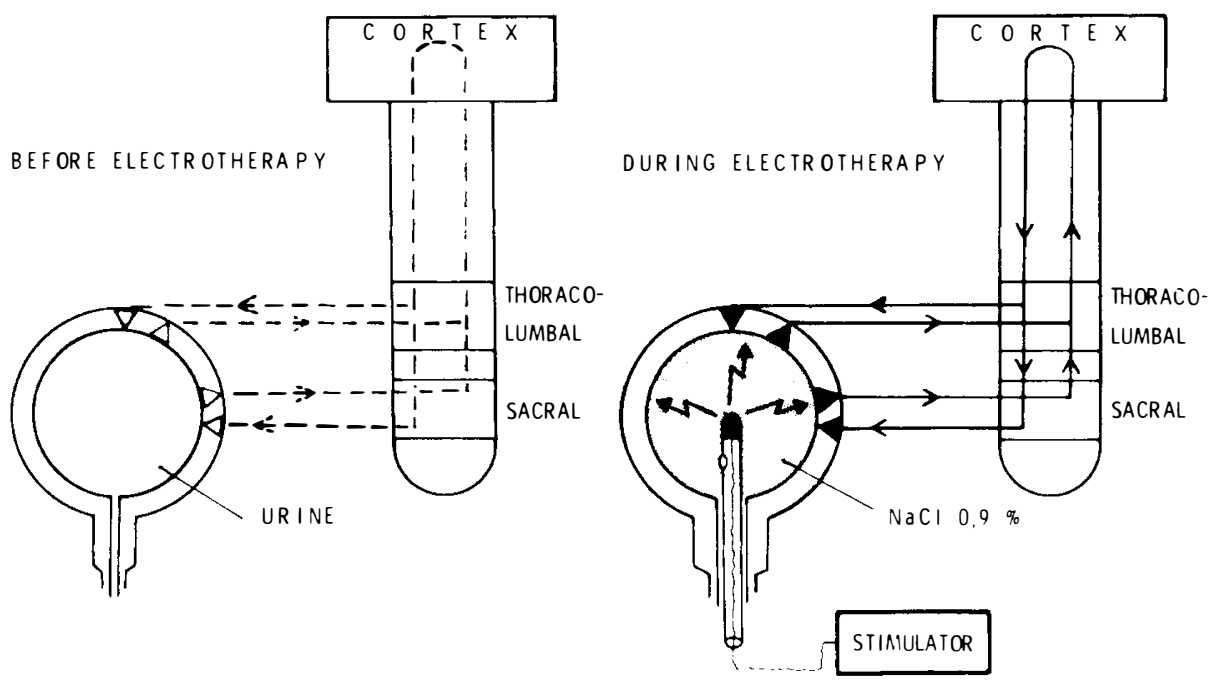

Figure 5 The working concept of the intravesical electrotherapy is based on the activation of the specific receptors in the bladder wall. The first step is the activation of the intramural motor system by receptor depolarisation; as soon as this local motor reaction reaches a certain strength activation of the micturition reflex takes place with increased sensation for urge and improved detrusor contractility (for further explanation see text).

\section{Conclusions}

Based on the urodynamic evaluation of detrusor and sphincter dysfunction, a rather simple but functional classification of the so called neuropathic bladder is presented. It has proved to be useful in clinical practice to detect risk factors and to plan treatment strategies.

The good experience with CIC for long term treatment of bladder emptying, the fact that detrusor hyperreflexia does respond to a new generation of spasmolytic (mainly anticholinergic) drugs, increasing experience with the artificial urinary sphincter in an improved design as well as with intravesical electrotherapy, and the so far excellent results with the sacral anterior root stimulator combined with sacral posterior root rhizotomy are responsible for changing concepts in the treatment of neurogenic detrusor-sphincter dysfunction. Current treatment strategies of four prototypes of neurogenic detrusor-sphincter dysfunction are presented and discussed.

This increased therapeutic armentarium offers more and more alternatives for the same type of neuropathic bladder with regard to the needs of individual patients.

The basis for the application of these new therapeutic measures remains adequate primary care during the spinal shock phase, and avoiding infection, bladder overdistension and urethral damage. Moreover, also with these new therapeutic strategies detrusor and sphincter dysfunction in these patients will not remain in a static state and functional and morphological changes may occur at any time, making lifelong urological care mandatory. 


\section{References}

BRAMBLE FJ 1982 The treatment of adult enuresis and urge incontinence by enterocystoplasty. British Fournal of Urology 54:693-696.

BRENDler ChB, Radebaugh LC, Mohler JL 1988 Topical oxybutynin chloride (Ditropan) for relaxation of dysfunctional bladder. Fournal of Urology 139(4):334 A.

BRINDley GS, Polkey CE, Rushton DN, CARDOZo L 1984 Sacral anterior root stimulators for bladder control in paraplegia: the first 40 cases. Proceedings of the International Society 14th Annual Meeting, Plattner-Druck, Innsbruck, pp 53-54.

GutTMANN L 1947 Spinal cord injuries: Discussion on the treatment and prognosis of traumatic paraplegia. Proceedings of the Royal Society of Medicine 40:219-225.

HILl VB, DAviES WE 1988 A swing to intermittent clean self-catheterization as a preferred mode of management of the neuropathic bladder for the dextrous spinal cord patient. Paraplegia 26:405412 .

Iwatsubo E, Wozumi J, Ando S, Kumazawa J 1988 Overdistension therapy of the bladder in paraplegics: 4 years follow-up. Handbook of 27th Annual Scientific Meeting, IMSOP, Perth 1988, p 67.

JILG G, MADERSBACHER H 1989 Control of detrusor hyperreflexia by intravesical application of Oxybutynin-Hydrochloride. Abstracts Scientific Meeting, IMSOP, Rome, 4-6 May 1989 (in press).

Jones RF, Burke D, Marosszeky JE, Giles JD 1970 A new agent for the control of spasticity. Fournal of Neurology, Neurosurgery and Psychiatry 33:464-470.

KATONA F 1958 Electric stimulation in diagnosis and therapy of bladder paralysis. Orvosi Hetilap 99:277-286.

KATONA F 1975 Stages of vegetative afferentation in reorganisation of bladder control during electrotherapy. Urologia Internationalis 30:19.

KrANE RJ, Siroky MB 1979 In: KrANE RJ, Siroky MB (eds) Clinical Neuro-Urology, Little, Brown and Company, Boston, pp 149-150.

MADERSBACHER H, SCOTT BF 1976 The twelve o'clock sphincterotomy: technique, indications, results. Paraplegia 14:261-267.

MADERSBACHER H, WEISSTEINER G 1977 Intermittent self-catheterization, an alternative in the treatment of neurogenic urinary incontinence in women. European Urology 3:82-84.

MADERSBACHER H 1977 The neuropathic urethra: urethrogram and pathophysiologic aspects. European Urology 3:321-332.

MAdersbacher H, Pauer W, Reiner E 1982 Rehabilitation of micturition by transurethral electrostimulation of the bladder in patients with incomplete spinal cord injuries. Paraplegia 20:19]-195.

MADERSBACHER H 1984 Blasen(re)habilitation mit neurogener Harnentleerungsstörung mittels Biofeedback unter Verwendung der transurethralen Elektrostimulation. Aktuel Urologie 15:248253.

Madersbacher H, Hetzel H, Gottinger F, Ebener A 1987 Rehabilitation of micturition in adults with incomplete spinal cord lesions by intravesical electrotherapy. Fournal of Neurourology and Urodynamics 6:230-232.

MCGUIRE E 1987 Personal communication.

Mounzer AM, Zeineh SJ, Lue TF, Schmidt RA, TANAgho EA 1984 Reversible pharmacological urethral sphincterotomy. Proceedings ICS, 14th Annual Meeting, Innsbruck. Buch- und Offsetdruck Plattner KG, Innsbruck, Austria, p 202.

Ross JC, DAMANSKI M 1953 Pudendal neurectomy in treatment of bladders in spinal injury. British Fournal of Urology 25:45-50.

Schmidbauer ChP, Chiang H, Raz Sh 1988 Three stage surgical treatment for severe female urinary incontinence. Fournal of Urology 139(4):391 A.

STEPHENSON TP, MUNDY AR 1985 Treatment of the neuropathic bladder by enterocystoplasty and selective sphincterotomy or sphincter ablation and replacement. British fournal of Urology 57:2731 